\title{
Abdominal angiostrongyliasis can be diagnosed with a immunochromatographic rapid test with recombinant galactin from Angiostrongylus cantonensis
}

\author{
Carlos Graeff-Teixeira ${ }^{1 /}{ }^{+}$, Vanessa Fey Pascoal ${ }^{2}$, Rubens Rodriguez ${ }^{3}$, \\ Alessandra Loureiro Morassutti ${ }^{3}$, Pewpan M Intapan ${ }^{4}$, Wanchai Maleewong ${ }^{4}$ \\ ${ }^{1}$ Universidade do Espírito Santo, Centro de Ciências da Saúde, Departamento de Patologia e Núcleo de Doenças Infecciosas, \\ Vitória, ES, Brasil \\ 2Pontifícia Universidade Católica do Rio Grande do Sul, Escola de Ciências da Saúde, Porto Alegre, RS, Brasil \\ ${ }^{3}$ Instituto de Patologia, Passo Fundo, RS, Brasil \\ ${ }^{4}$ Khon Kaen University, Faculty of Medicine and Mekong Health Science Research Institute, Department of Parasitology \\ and Excellence in Medical Innovation, and Technology Research Group, Khon Kaen, Thailand
}

Angiostrongylus costaricensis is the causative agent of abdominal angiostrongyliasis, a zoonotic infection that may produce severe eosinophilic enterocolitis or hepatitis in humans. Parasites are usually not released in stools and serology has an important role in diagnosis. Since cross-reactivity is demonstrated between A. costaricensis and another metastrongylid worm, A. cantonensis, we tested heterologous recombinant galectin as a probe in an immunochromatographic rapid diagnostic test (ICT-RDT) for detection of anti-A. costaricensis antibodies. Almost all (11/12) positive control sera from A. costaricensis infected patients were positive at ICT RDT. These are preliminary indications that r-galectin ICT-RDT is useful for diagnosing A. costaricensis infection.

Key words: angiostrongyliasis - eosinophilic enterocolitis - eosinophilic hepatitis

Abdominal angiostrongyliasis (AA) is caused by $\mathrm{An}$ giostrongylus costaricensis, an intra-arterial nematode from Mestastrongyloidea superfamily. The worms live inside mesenteric arteries in rodents, their first stage larvae (L1) are released in feces and develop into third stage larvae (L3) in fibromuscular tissues of terrestrial mollusks. (1) L3 is infective for vertebrates and transmission occurs through ingestion, including man as an accidental host. The human infection may manifest as an eosinophilic and granulomatous enterocolitis and hepatitis. ${ }^{(2)}$ Abdominal angiostrongyliasis have been detected in most countries in Americas, from southern Unites States to northern Argentina. There are indications that most of the infections are oligosymptomatic or without any clinical manifestations. ${ }^{(3)}$ Only a few patients present with complicated acute abdomen syndromes requiring surgical treatment, especially for resection of necrotic and/or thickened intestinal segments. Since notification is not mandatory, there are no precise incidence estimates, but a reference pathological laboratory (Institute of Pathology, Passo Fundo) in the endemic area in Brazil's southernmost State, Rio

doi: 10.1590/0074-02760200201

Financial support: This study was co-supported by Distinguished Research Professor Grant, Thailand Research Fund (TRF) (grant no. DPG6280002), and Khon Kaen University (PMI, WM).

CG-T is a recipient of a CNPq-Brazil 302675/2019-1 fellowship.

+ Corresponding author: graeff.teixeira@gmail.com

(D) https://orcid.org/0000-0003-2725-0061

Received 28 April 2020

Accepted 20 August 2020
Grande do Sul (28 $8^{\circ} 15^{\prime} 46^{\prime \prime} \mathrm{S}$; 52 $\left.2^{\circ} 24^{\prime} 24^{\prime \prime} \mathrm{W}\right)$, have diagnosed AA approximately three times per year in the years 2014-2018 (Unpublished observations).

Another metastrongylid nematode causing human disease is $A$. cantonensis. With a biological cycle similar to $A$. costaricensis, but distinguished by the final habitat for worms in the pulmonary arteries and development of larvae in transit through cerebral tissues leading to eosinophilic meningitis. ${ }^{(4)} A$. cantonensis originally came from southeastern Asian countries and Islands in the Pacific Ocean and has spread across many countries. ${ }^{(5)}$

Parasitological diagnosis is not an option in angiostrongyliasis, since larvae in feces was never consistently detected in AA patients and only rarely documented in cerebrospinal fluid in eosinophilic meningitis. ${ }^{(6)}$ Development of immunological methods have faced two main difficulties: (i) large production of antigens is laborious and it depends on in vivo maintenance of parasites, especially for $A$. costaricensis, which shows less than optimal adaptability to laboratory mice strains; ${ }^{(7)}$ (ii) lack of a large reference panel of sera with precise characterisation of true-positive samples by histopathology. Complicated abdominal angiostrongyliasis leading to biopsy or anatomopathological examination and identification of the parasite is not frequent. ${ }^{(3)}$ Moreover, central nervous system lesions produced by $A$. cantonensis are usually not investigated with biopsy and larvae are seldom detected in cerebrospinal fluid preventing etiological confirmation. ${ }^{(6)}$

Tissue dwelling parasites, especially those that do not complete their cycle, like angiostrongylid worms in humans, may not release any parasitic structure in body fluids or excreta. Therefore, serology stands as the main diagnostic tool. Crude worm antigens were employed in 
a Latex agglutination test in use in Costa Rica for decades. Eggs and small peptides were preliminarily assayed, but not applied in routine diagnosis. ${ }^{(8,9)}$

Detection of serum IgG in an immunoenzymatic assay (IgG-ELISA) employing crude female $A$. costaricensis worm antigens ${ }^{(10)}$ was standardised for diagnosis of abdominal angiostrongyliasis. Crude female antigens from A. cantonensis was later tested and replaced A. costaricensis antigens, in order to overcome the difficulties to obtain antigenic preparations. ${ }^{(11)}$ Use of heterologous antigens, i.e. cross-reacting antigens coming from filogenetically close species or from other sources, has been investigated for other helminthiasis diagnosis. One example is keyhole limpet haemocianin (KHL), a molecule sharing carbohydrate moieties cross-reacting with surface molecules on young Schistosoma spp. worms. ${ }^{(12)}$ Another example is Schistosoma mansoni antigens for diagnosing urinary schistosomiasis, caused by $S$. hematobium . (13) Cross-reactivity between Angiostrongylus congeneric species have been demonstrated: anti-A. cantonensis antibodies recognises $A$. costaricensis antigens ${ }^{(14)}$ and vice versa, sera from $A$. costaricensis infected patients also recognise crude $A$. cantonensis antigens. ${ }^{(11)}$

Immunological diagnosis of $A$. cantonensis infection is far more advanced than with abdominal angiostrongyliasis. ${ }^{(5,15,16,17)}$ After a recent standardisation of a rapid diagnostic test (RDT) for IgG antibody detection through a immunochromatographic kit (RDT-ICT) using a recombinant galectin antigen from $A$. cantonen$s i{ }^{(18)}$ a collaboration study was established to verify the sensitivity of RDT-ICT to detect anti-A. costaricensis antibodies. Galectins are a class of proteins that bind to N-glycans and Galactose $\beta 1-4$ fucose is one target already identified in nematodes, including Ascaris suum and other helminthes of veterinary importance. ${ }^{(19)}$ Since ascariasis is a prevalent human infection in many areas, the possibility of antibody cross-reactivity between galectins from angiostrongylid and ascarid worms is to be addressed in future specificity evaluations.

A batch of 20 tests was sent from Khon Khaen University in Thailand for this preliminary evaluation with sera from individuals with confirmed (12 samples) and suspected (four samples) diagnosis of abdominal angiostrongyliasis with objective of evaluating the efficacy of r-galectin as a probe to capture anti- $A$. costaricensis antibodies. The samples were sent for routine serology and the patients gave informed consent at the time of collection for anonymously storage in the serum reference panel for angiostrongyliasis (according to Brazilian regulations and with the Helsinki Declaration of 1975, as revised in 1983; the protocol was approved by PUCRS ethics committee CEP 01/96).

TABLE

Ratio values for anti-Angiostrongylus costaricensis IgG enzyme-linked immunosorbent assay (ELISA), band intensities obtained in a recombinant galectin immunochromatographic (ICT) rapid diagnostic test (RDT) and histopathological findings defining 12 true-positive samples and four suspected abdominal angiostrongyliasis samples from southern Brazil, 2014 to 2018. ICT-RDT is considered positive with band intensities higher than 0.5

\begin{tabular}{|c|c|c|c|}
\hline IgG-ELISA ratio & ICT-RDT Band intensity & ICT-RDT result & Histopathology findings \\
\hline \multicolumn{4}{|c|}{ True-positive samples } \\
\hline 11.55 & 0.5 & negative & worms \\
\hline 5.62 & 1 & positive & worms \\
\hline 1.22 & 1 & positive & worms \\
\hline 51.5 & 2 & positive & worms \\
\hline 17.4 & 2 & positive & eggs \\
\hline 4.74 & 2 & positive & worms \\
\hline 3.4 & 3 & positive & worms \\
\hline 2.94 & 3 & positive & worms \\
\hline 1.3 & 3 & positive & worms \\
\hline 15 & 4 & positive & worms \\
\hline 19.05 & 4 & positive & eggs \\
\hline 1.07 & 4 & positive & eggs \\
\hline
\end{tabular}

Suspected abdominal angiostrongyliasis

\begin{tabular}{lllc}
\hline 4.58 & 0 & negative & eosinophilia \\
\hline 1.43 & 1 & positive & eosinophilia \\
\hline 2.52 & 2 & positive & eosinophilia \\
1.07 & 3 & positive & necrosis \\
\hline
\end{tabular}


Immunochromatographic RDT was performed according to manufacturers' instructions (Kestrel BioSciences Co., Pathumthani, Thailand). Briefly, $5 \mu \mathrm{L}$ of diluted serum samples (1:30) was filled in a sample well, followed by $90 \mu \mathrm{L}$ of running buffer. The result was presented through visual examination within $10 \mathrm{~min}$ after the addition of the running buffer. If red bands appeared at both $\mathrm{T}$ and $\mathrm{C}$ lines, the result was positive and if a red band appeared only at the $\mathrm{C}$ line, the result was negative. The band intensity was interpreted visually according to the reference board (with 0.5 as the cut-off level). The RDT-ICT kits that revealed T line intensities greater than the cut-off point were regarded as positive results. Pooled positive and negative reference sera were produced by mixing equal volumes of Thai human serum samples from 10 eosinophilic meningitis or meningoencephalitis and clinical suspected angiostrongyliasis cantonensis patients and 10 normal healthy volunteers, respectively and were also used for testing as internal controls.

IgG-ELISA was performed as routine diagnostic method at the Molecular Parasitology Laboratory, Pontifícia Universidade Católica do Rio Grande do Sul, Porto Alegre, Brazil, with accuracy estimates of $88 \%$ sensitivity and $78 \%$ specificity. ${ }^{(1)}$ Briefly, polystyrene plates were sensitised with $0.5 \mu \mathrm{g}$ of antigen per well and test sera were diluted at 1:200.

Except for one sample with a 0.5 degree line intensity, 11 out of 12 (91.6\% sensitivity, 90.41-92.91, 95\% confidence interval) positive controls serum samples from patients with identification of adult worms or eggs had positive detection of antibodies by the RDT, with an even distribution of intensities from 1.0 to 4.0 (Table). Reactivity of these same sera was tested by IgG-ELISA with ratios of positivity from 1.07 to 51.5 and a very low coefficient of correlation: 0.19288 . The only positive control serum had an ELISA ratio of 11.5. It is important to note that ELISA employs a very complex set of proteins while RDT-ICT has a unique recombinant protein, what may explain lack of correlation between ELISA ratios and intensity of bands in RDT-ICT. From four samples without identification of parasitic structures in histopathological examination, but with a suspected diagnosis based on histopathological criteria, three were RDT-ICT positive with degrees of intensity: one, two and three.

In conclusion, these are preliminary indications that RDT-ICT with heterologous r-galectin from A. cantonensis is useful for serological diagnosis of abdominal angiostrongyliasis in individuals presenting suggestive clinical and laboratorial findings. While A. cantonensis infections manifest as meningitis, $A$. costaricensis causes intestinal or hepatic pathology, what allows the differential etiological diagnosis in clinical settings. In areas with occurrence of both parasites, serology is not an adequate tool for prevalence studies, because of the extensive cross-reactivity already mentioned. ${ }^{(11,14)}$ A prospective multicentric longitudinal study of accuracy of RDT-ICT in A. costaricensis endemic areas, including a well-characterised negative control panel for specificity evaluation is needed. The new diagnostic tool shall contribute both to epidemiological surveys and better management of patients with abdominal angiostrongyliasis.

\section{AUTHORS' CONTRIBUTION}

CG-T, PMI and WM - Conception and design; VFP and ALM - acquisition, analysis and interpretation of data; CG-T drafting; CG-T, VFP, ALM, PMI and WM - revision and final version. All authors agreed to be accountable for all aspects of the work in ensuring that questions related to the accuracy or integrity of any part of the work are appropriately investigated and resolved. The contents of this report are solely the responsibility of the authors and do not necessarily represent the official views of the TRF and the KKU.

\section{REFERENCES}

1. Morera P. Life history and redescription of Angiostrongylus costaricensis Morera and Céspedes, 1971. Am J Trop Med Hyg. 1973; 22(5): 613-21.

2. Graeff-Teixeira C, Camillo-Coura L, Lenzi HL. Histopathological criteria for diagnosis of abdominal angiostrongyliasis. Parasitol Res. 1991; 77(7): 606-11.

3. Graeff-Teixeira C, Goulart AH, Brum CO, Laitano AC, Sievers-Tostes C, Zanini GM, et al. Longitudinal clinical and serological survey of abdominal angiostrongyliasis in Guaporé, southern Brazil, from 1995 to 1999. Rev Soc Bras Med Trop. 2005; 38(4): 310-5.

4. Alicata JE. The discovery of Angiostrongylus cantonensis as a cause of human eosinophilic meningitis. Parasitol Today. 1991; 7(6): 151-3.

5. Barratt J, Chan D, Sandaradura I, Malik R, Spielman D, Lee $\mathrm{R}$, et al. Angiostrongylus cantonensis: a review of its distribution, molecular biology and clinical significance as a human pathogen. Parasitology. 2016; 143(9): 1087-118. doi: 10.1017/ S0031182016000652.

6. Kuberski T, Bart RD, Briley JM, Rosen L. Recovery of Angiostrongylus cantonensis from cerebrospinal fluid of a child with eosinophilic meningitis. J Clin Microbiol. 1979; 9(5): 629-31.

7. Garrido CT, Morassutti AL, Barradas JRS, Graeff-Teixeira C. Evaluating host-parasite co-adaptation relationships involving Angiostrongylus costaricensis. J Helminthol. 2017; 93(1): 76-80. https://oi.org/10.1017/ S0022149X1700116X.

8. Bender AN, Maurer RL, Silva MCF, Ben R, Terraciano PB, Silva ACA, et al. Eggs and reproductive organs of female Angiostrongylus costaricensis are more intensely recognized by human sera from acute phase in abdominal angiostrongyliasis. Rev Soc Bras Med Trop. 2003; 36(4): 449-54.

9. Abraham ES, Schultz-Key H, Geiger SM. Caracterización de antígenos de bajo peso molecular de Angiostrongylus costaricensis, reconocidos durante una infección experimental en roedores. Parasitol Latinoam. 2004; 59(1): 8-13.

10. Geiger SM, Laitano AC, Sievers-Tostes C, Agostini AA, SchulzKey H, Graeff-Teixeira C. Detection of the acute phase of abdominal angiostrongyliasis with a parasite-specific IgG enzyme linked immunosorbent assay. Mem Inst Oswaldo Cruz. 2001; 96(4): 515-8.

11. Ben R, Rodriguez R, Agostini AA, Graeff-Teixeira C. Use of heterologous antigens for the immunodiagnosis of abdominal angiostrongyliasis by an enzyme-linked immunosorbent assay. Mem Inst Oswaldo Cruz. 2010; 105(7): 914-7.

12. Beck L, Van-Lume DS, Souza JR, Domingues ALC, Favre T, Abath FGC, et al. Discriminating acute from chronic human schistosomiasis mansoni. Acta Trop. 2008; 108(2-3): 229-33.

13. van Gool T, Vetter H, Vervoort T, Doenhoff MJ, Wetsteyn J, Overbosch D. Serodiagnosis of imported schistosomiasis by a combination of a commercial indirect hemagglutination test with Schistosoma mansoni adult worm antigens and an enzyme-linked immunosorbent assay with $S$. mansoni egg antigens. J Clin Microbiol. 2002; 40(9): 3432-7. 
14. Dekumyoy P, Komalamisra C, Nuamtanong S, Nacapunchai D, Sinnawong M, Shanaha P, et al. Angiostrongyliasis: analysis of antigens of Angiostrongylus costaricensis adult worms versus IgG from infected patients with Angiostrongylus cantonensis. Southeast Asian J Trop Med Public Health. 2000; 31(Suppl. 1): 48-53.

15. Eamsobhana P, Yong HS. Immunological diagnosis of human Angiostrongylus cantonensis (Nematoda: Angiostrongylidae). Int $\mathrm{J}$ Infect Dis. 2009; 13(4): 425-31.

16. Veríssimo CM, Morassutti AL, von Itzstein M, Sutov G, HartleyTassell L, McAtamney S, et al. Characterization of the N-glycans of female Angiostrongylus cantonensis worms. Exp Parasitol. 2016; 166(2): 137-43. https://doi.org/10.1016/j.exppara.2016.04.012.
17. Cognato BB, Handali S, Morassutti AL, Silva AJ, Graeff-Teixeira C. Heterologous expression of three antigenic proteins from $\mathrm{An}$ giostrongylus cantonensis: ES-7, Lec-5, and 14-3-3 in mammalian cells. Mol Biochem Parasitol. 2018; 221(1): 32-5. https://doi. $\operatorname{org} / 10.1016 /$ j.molbiopara.2018.03.001.

18. Somboonpatarakun C, Intapan PM, Sadaow L, Rodpai R, Sanpool O, Maleewong W. Development of an immunochromatographic device to detect antibodies for rapid diagnosis of human angiostrongyliasis. Parasitology. 2020; 147(2): 194-8. https://doi. org/10.1017/S0031182019001495.

19. Takeuchi T, Arata Y, Kasai K. Galactose $\beta 1-4$ fucose: a unique disaccharide unit found in N-glycans of invertebrates including nematodes. Proteomics. 2016; 16(24): 1337-47. doi 10.1002/ pmic. 201600001 . 